\title{
SOBRE EL COLAPSO DE LAS ESTRUCTURAS MATEMÁTICAS Y FÍSICAS EN EL REALISMO ESTRUCTURAL ÓNTICO*
}

\author{
Cristian Soto** \\ http://orcid.org/0000-0001-5675-8943 \\ cssotto@uchile.cl
}

RESUMEN La sección 1 introduce lo que llamo la tesis del colapso de las estructuras matemáticas y las estructuras físicas. La sección 2 examina si acaso la indispensabilidad de las matemáticas para la física fundamental involucra la adopción del platonismo matemático, en este caso acerca de estructuras matemáticas, como argumenta el realismo estructural óntico. La sección 3 muestra que la adopción de la tesis del colapso arriesga introducir la hipótesis del universo matemático. Desde la perspectiva de la concepción inferencial en filosofía de las matemáticas aplicadas, la sección 4 examina aspectos epistémicos del rol de las matemáticas en la teorización científica, derivando distinciones entre estructuras matemáticas y estructuras físicas. El argumento concluye en la sección 5 indicando algunos límites para la presente estrategia.

Palabras clave Estructuras matemáticas, estructuras físicas, realismo estructural óntico, hipótesis del universo matemático, platonismo matemático, concepción inferencial de las matemáticas aplicadas.

* Artigo submetido em 16/05/2018 e aprovado em 04/02/2019. El presente artículo es resultado de investigación del proyecto FONDECYT de Iniciación 11160324, The Physico-Mathematical Structure of Scientific Laws: On the Roles Mathematics, Models, Measurements and Metaphysics in the Construction of Scientific Laws, CONICYT, Chile. Investigador responsable: Dr. Cristian Soto.

** Universidad de Chile - Departamento de Filosofía. Santiago de Chile, Chile. 
ABSTRACT Section 1 introduces what I call the collapse thesis, which holds that the boundaries of mathematical and physical structures blur or even vanish in certain areas of fundamental physics. Section 2 examines the claim that the indispensability of mathematics to fundamental physics requires the adoption of mathematical Platonism, this time concerning the reality of mathematical structures, as ontic structural realism contends. Section 3 demonstrates that the collapse thesis introduces the threat of the mathematical universe hypothesis. Then, from the perspective of the inferential conception in the philosophy of applied mathematics, section 4 examines epistemic features of the role of mathematics in scientific theorizing, drawing distinctions between mathematical and physical structures. Lastly, the argument closes in section 5 pointing out some of the limits of the proposed strategy for a distinction.

Keywords Mathematical structures, physical structures, ontic structural realism, mathematical universe hypothesis, mathematical Platonism, the inferential conception of applied mathematics.

\section{1. ¿Qué es la tesis del colapso?}

El debate reciente en torno a la viabilidad del realismo estructural óntico se ha desarrollado en diversas direcciones. Algunos de sus defensores sostienen una tesis radical acerca de las estructuras matemáticas y las estructuras físicas, que llamaremos la tesis del colapso, a saber: áreas de la física fundamental sugieren que no hay una distinción entre las estructuras matemáticas y las estructuras físicas, invitándonos a asumir que ellas ostentan el mismo estatus ontológico. Esta tesis involucra, entre otras cosas, que el problema que algunos intentan resolver al separar las unas de las otras es un pseudoproblema, a tal punto que cualquier intento por delinear una distinción entre ellas consistiría en palabras vacías (Ladyman y Ross, 2007, pp. 159-169). ${ }^{1}$

Una de las razones que motiva la tesis del colapso dice relación con el rol indispensable que las matemáticas juegan en diversas ramas de la física fundamental. Las matemáticas proveen estructuras que facilitan adecuadamente la construcción de representaciones de estructuras físicas en áreas tales como la mecánica cuántica, la teoría de la relatividad y la teoría cuántica de campos.

1 Téngase en cuenta que no todas las versiones del realismo estructural óntico se comprometen en igual medida con la tesis del colapso. French (2014), por ejemplo, articula una versión del realismo estructural óntico que intenta morigerar el alcance de la tesis del colapso. 
Los límites entre las estructuras matemáticas y las estructuras físicas se tornan difusos en estos niveles de teorización física, especialmente considerando que las estructuras matemáticas nos ofrecen nuestro mejor acceso a estos dominios de la realidad (French y Ladyman, 2003; Ladyman y Ross, 2007). Ante esto, el realismo estructural óntico sugiere la adopción de una forma de platonismo matemático que postule la realidad de las estructuras matemáticas, y que a la vez vaya de la mano con el tipo de realismo naturalista que defiende que la naturaleza de la realidad es estructural en su nivel fundamental (Ladyman y Ross 2007, p. 237). La discusión en esta dirección ha llegado más lejos aún. French (2014), en particular, advierte que la falta de distinción entre las estructuras físicas y las matemáticas podría conducirnos a la adopción de la hipótesis del universo matemático, que es la idea que sostiene que la realidad es en última instancia una estructura matemática omniabarcante.

En el presente artículo, me interesa mostrar que no tenemos que asumir la tesis del colapso, como lo sugiere el realismo estructural óntico en la versión de Ladyman y Ross (2007). Argumentaré que tal idea descansa en una confusión respecto de consideraciones ontológicas y epistémicas acerca del rol de las estructuras matemáticas en la teorización científica. El hecho de que la representación de estructuras físicas requiera de estructuras matemáticas abstractas, que bien podrían resultar indispensables, no nos fuerza a asumir que la estructura física misma que se quiere representar tenga que ser abstracta. French (2014, p. 17) ofrece un comentario similar en esta dirección. Sin embargo, a ello cabe todavía añadir que la indispensabilidad de las estructuras matemáticas abstractas para la representación de estructuras físicas concretas no entraña la idea de que las primeras gocen de un estatuto ontológico similar a las segundas. Las consideraciones epistémicas dicen relación con cuestiones concernientes a la manera en la cual las comunidades científicas construyen representaciones de sistemas físicos en términos del lenguaje de las matemáticas. Tiene que reconocerse, no obstante, que tales consideraciones epistémicas son insuficientes para justificar la adopción de la tesis ontológica que sugiere la fusión de las estructuras matemáticas y las físicas, adoptando un compromiso ontológico similar con ellas.

En breve, en lo que sigue mostraré que no contamos con evidencia para equiparar el estatuto ontológico de las estructuras matemáticas y las físicas, aun cuando en algunos escenarios en física fundamental no podamos tener acceso a estructuras físicas sin emplear estructuras matemáticas. En particular, recurriré a herramientas provistas por la filosofía de las matemáticas aplicadas, particularmente por elaboraciones recientes de la concepción inferencial de la aplicación de estructuras matemáticas a estructuras físicas (Bueno y Colyvan, 
2011; Bueno y French, 2018) con el objetivo de trazar distinciones entre ellas. Mostraré que la concepción inferencial nos ofrece un marco metafísicamente deflacionario que nos permite aclarar la distinción en cuestión en vistas del rol que juegan estos tipos de estructuras en la teorización científica.

\section{La tesis del colapso}

El realismo estructural óntico defiende que "el mundo tiene una estructura modal objetiva que es ontológicamente fundamental, en el sentido de que no superviene de las propiedades intrínsecas de un conjunto de individuos" (Ladyman y Ross, 2007, p. 130). Se sostiene que la realidad está compuesta de estructuras descritas por las diversas áreas de la física fundamental, las cuales no descansan en individuos preexistentes. Una de las motivaciones tras esta tesis consiste en que ella busca responder a la necesidad de una ontología que dé cuenta de las dificultades que emergen en las interpretaciones filosóficas de la física fundamental, especialmente en mecánica cuántica, en donde el principio de la identidad de los indiscernibles parece no aplicarse a los estados de entrañamiento cuántico que involucran dos sistemas no individuables (Ladyman, 1998). El realismo estructural óntico sugiere que todo lo que parece haber en estados de entrañamiento cuántico son estructuras modales ontológicamente fundamentales, las cuales se asume que son previas a los individuos a las que ellas dan lugar.

¿Cómo se distinguen las estructuras matemáticas y las estructuras físicas dentro de este marco ontológico? El realismo estructural óntico se niega a ofrecer una respuesta, sosteniendo que cualquier intento en esta dirección "equivaldría a palabras vacías y se aventuraría más allá de lo que permite el PCN [principio de cierre naturalista]" (Ladyman y Ross, 2007, p. 158). En la sección 3 señalaremos la definición del principio del cierre naturalista. Por lo pronto, nótese que el hecho de que se decline ofrecer una respuesta a la pregunta sobre la distinción entre estructuras matemáticas y estructuras físicas ha sido considerado una "evasión intelectual" (Humphreys, en Stanford et al. 2010, p. 172). En breve, se nos pide que creamos que las estructuras constituyen el fundamento ontológico modal de la realidad, pero no se aclara si estas estructuras son físicas o matemáticas, o una mezcla de ellas.

Una de las razones por las cuales el realismo estructural óntico sostiene la tesis del colapso de las estructuras matemáticas y las físicas dice relación con la contribución epistémica de las matemáticas a las ciencias, y a la física fundamental en particular. Se sostiene, entre otras cosas, que el hecho de que nosotros, como especie, hayamos aprendido tanto a representar el mundo como 
a razonar matemáticamente facilitó la tarea de lograr conocimiento acerca de aspectos objetivos de la realidad (Ladyman y Ross, 2007, p. 2). Según el realismo estructural óntico, uno de los beneficios de la formulación matemática de las teorías científicas consiste en ayudar a esclarecer compromisos ontológicos. Considérese el siguiente pasaje:

\begin{abstract}
Si a uno se le pidiera que represente la ontología del mundo de acuerdo, por ejemplo, a la RG [relatividad general], uno presentaría el aparato de la geometría diferencial y las ecuaciones de campo, y luego continuaría explicando la topología y otras características de modelos particulares [...] de estas ecuaciones, las cuales se cree que describen el mundo real (Ladyman y Ross, 2007, p. 159).
\end{abstract}

Este pasaje refleja un aspecto central de algunas teorías fundamentales según el realismo estructural óntico, a saber: se emplean estructuras matemáticas para representar relaciones estructurales modales últimas. Sin embargo, los problemas que involucra la tesis del colapso para la defensa del realismo estructural óntico comienzan cuando se sostiene que la representación matemática es indispensable para las ciencias (Ladyman y Ross 2007, p. 159). La tesis de la indispensabilidad de las matemáticas para la práctica científica ha sido discutida en detalle en la literatura reciente (Soto, 2019). Aunque algunos han considerado que el argumento de la indispensabilidad es uno de los elementos claves de la defensa del platonismo matemático (Colyvan, 2001), otros han puesto en cuestión el rol indispensable de las matemáticas en la construcción de teorías científicas. Los argumentos a este último respecto provienen tanto de las ciencias como de la filosofía de las ciencias (Feynman, 1965; Field, 1980 y 1989; Pincock, 2011). Hasta donde sabemos, las matemáticas - sobre todo aquellas que constituyen estructura matemática excedente (surplus mathematical structure, en la jerga de Redhead, 1975), y que aparecen en la construcción de idealizaciones de sistemas físicos a los que no tenemos acceso epistémico robusto - podrían ser eliminables en el curso de la investigación científica.

Además, la aparición de estructuras matemáticas en las teorías científicas no es un indicador inequívoco de compromiso ontológico o epistémico. Pincock (2011, p. 69), entre otros, ha argumentado que las matemáticas excedentes de una teoría pueden revelar ignorancia de los detalles de la constitución física de los mecanismos que queremos representar. Explorando el caso de la teoría maxwelliana del campo electromagnético, Pincock muestra que quienes profesan inclinación por la interpretación realista de la teorización científica leen las ecuaciones de Fresnel que reaparecen intactas en Maxwell como si ellas garantizaran la continuidad ontológica en escenarios de cambio teórico 
(Worrall, 1989). ${ }^{2}$ Por el contrario, en tales casos cabe esperar que el componente matemático de las teorías sea eliminado con el progreso de la ciencia cuando el mecanismo responsable de los fenómenos electromagnéticos sea finalmente descubierto.

Una vez asumida la indispensabilidad de las matemáticas para la investigación científica, el realismo estructural óntico defiende la posibilidad de eliminar la distinción entre las estructuras matemáticas y las estructuras físicas. En esta dirección, se nos invita "abandonar la distinción entre las estructuras abstractas empleadas en el modelamiento y las estructuras concretas que son los objetos de la física" (Ladyman y Ross, 2007, p. 159). Con ello se da un primer paso para comprometerse con una forma de platonismo matemático que pueda trabajar mano a mano con el realismo naturalista.

Según ello, la práctica científica nos ofrecería evidencia para defender la tesis del colapso. Primero, la distinción entre estructuras físicas y estructuras matemáticas se habría llevado a cabo tradicionalmente en términos de poderes causales y localización espacio-temporal. Pero los fenómenos cuánticos no responden a tales criterios: no resulta evidente de suyo que los estados de entrañamiento cuántico estén causalmente relacionados o localizados espaciotemporalmente de la manera en la que la mecánica clásica describe los fenómenos en un contexto clásico. Y segundo, la dependencia teórica que evidencia la física fundamental respecto de idealizaciones matemáticas, tales como los espacios de Hilbert, socavaría la relevancia de la distinción entre lo abstracto y lo concreto en la teorización científica (Ladyman y Ross, 2007, p. 160). En consecuencia, la interpretación realista de las teorías científicas carecería de criterios para distinguir entre compromisos ontológicos con estructuras matemáticas y estructuras físicas, si ambas resultan igualmente indispensables.

La conclusión que deriva el realismo estructural óntico a partir de estos análisis es la siguiente:

Solamente estaremos interesados en razones para adoptar un platonismo que sea compatibles con el PCN [principio del cierre naturalista]. Una posibilidad clara y muy interesante es que en la medida en que lleguemos en verdad a acostumbrarnos a pensar en el universo físico en términos de patrones más bien que cosas pequeñas, se reducirá o incluso desaparecerá la separación tradicional entre el realismo platónico

2 Cabe destacar que formulaciones similares de este argumento usualmente asociado a Worrall (1989) han sido desarrolladas por Chiappin (1989 y 2018). Recuérdese, por lo demás, que el realismo estructural epistémico asevera que la conservación de estructuras matemáticas en transiciones de cambio teórico en ciencias constituye nuestra mejor apuesta para la defensa del realismo científico. Se trata, por cierto, de un tipo de realismo selectivo que respalda la interpretación realista de la teorización científica en la formalización matemática de las teorías científicas. Un examen de los límites del realismo estructural epistémico puede encontrarse en Psillos (1999). 
acerca de las matemáticas y el realismo naturalista acerca de la física (Ladyman y Ross 2007, p. 237).

En breve, la tesis del colapso descansa en el supuesto de que la contribución epistémica de las estructuras matemáticas a la construcción de representaciones de estructuras físicas es indispensable. A partir de ello, se sostiene que los límites entre lo matemático y lo físico se oscurecen e incluso desaparecen. En la sección 3 argumentaré que una de las debilidades de la tesis del colapso consiste en que ella arriesga introducir la amenaza de la hipótesis del universo matemático. Retomaré en ese punto la cuestión de si acaso hay una forma de platonismo matemático que sea compatible con el principio del cierre naturalista. Luego, en la sección 4 argumentaré que es preciso insistir en separar con mayor claridad consideraciones epistémicas y ontológicas acerca del lugar y del rol de las estructuras matemáticas y las físicas en la teorización científica. Mostraré que el realismo estructural óntico aborda consideraciones epistémicas cuando reconoce que la física fundamental depende de las matemáticas a la hora de representar estructuras físicas fundamentales. Igualmente, otra consideración epistémica es aquella que apunta a la posibilidad de que nos lleguemos a acostumbrar a concebir el universo en términos de estructuras matemáticas que representan patrones reales. Sin embargo, a partir de tales consideraciones epistémicas, el realismo estructural óntico da el salto a conclusiones ontológicas.

\section{Desde la tesis del colapso a la hipótesis del universo matemático}

La hipótesis del universo matemático representa la culminación de la identidad entre las estructuras matemáticas y las físicas, sosteniendo que "nuestra realidad física externa es una estructura matemática” (Tegmark, 2014, p. 319). La hipótesis del universo matemático se compromete con las siguientes ideas: las estructuras matemáticas son eternas e inmutables; el espacio-tiempo existe en tales estructuras matemáticas; igualmente, los fenómenos que tienen lugar en el espacio-tiempo existen en tales estructuras matemáticas.

La pregunta que nos interesa a continuación es la siguiente: ¿nos conduce la tesis del colapso a la hipótesis del universo matemático? La respuesta a este respecto no es sencilla, puesto que, aun cuando puedan aducirse argumentos para separar la tesis del colapso y la hipótesis del universo matemático, ambas incluyen compromisos con la realidad de las estructuras matemáticas.

Entre las razones que podrían aducirse para separar la tesis del colapso y la hipótesis del universo matemático encontramos las siguientes. En primer lugar, Tegmark solamente refiere de paso al realismo estructural, limitándose a afirmar 
que la idea de que la realidad es una estructura matemática "corresponde a la versión 'óntica' del realismo estructural universal en la terminología filosófica" (Tegmark, 2008, p. 107). Sin embargo, no resulta claro a qué terminología filosófica se refiere en estas líneas, así como también resulta confuso si el realismo estructural universal tiene que ser identificado con el realismo estructural óntico.

En segundo lugar, el artículo seminal de Ladyman (1998) aparece mencionado una vez en una nota al pie de página en Tegmark (2008, p. 107, n. 13), sin que se ofrezca un análisis detallado al respecto, ni mucho menos que se consideren otras referencias a la literatura relevante. En su elaboración más exhaustiva de la hipótesis del universo matemático, Tegmark (2014) no presenta argumentos ulteriores a este respecto. Por el contrario, las referencias al realismo estructural óntico desaparecen, y se menciona únicamente el trabajo de Worrall (1989) sobre el realismo estructural en su variante epistémica en nota al pie de página (Tegmark 2014, p. 260). Podría aducirse entonces que el trabajo de Tegmark no ofrece razones para asimilar la tesis del colapso con la hipótesis del universo matemático.

En tercer lugar, una razón adicional que podría sugerirse es la siguiente: la hipótesis del universo matemático es afín al pitagoreanismo más bien que al platonismo. En este contexto, el pitagoreanismo matemático es la tesis que sostiene que la realidad está constituida en última instancia por entidades y estructuras matemáticas eternas e inmutables. Como tal, el pitagoreanismo matemático no solamente le otorga realidad a la ontología matemática, sino que también entraña la concepción que sostiene que la existencia de tal reino matemático descansa sobre fundamentos más firmes que la existencia del mundo físico.

Sin embargo, la referencia de Tegmark al platonismo radical suscita confusión. Aunque podría aceptarse que la afirmación de que "todas las estructuras matemáticas tienen el mismo estatus ontológico [que el mundo físico]" (Tegmark, 2008, p. 125) es compatible con el platonismo matemático, no se esbozan razones que nos permitan avanzar en esta dirección. Con una orientación pitagórica declarada, Tegmark acaba por concluir que "no solamente el espacio-tiempo es una estructura matemática, sino todo lo que encontramos en él [...], que la creación y la destrucción son ilusiones puesto que ellas involucran cambios; [y que] las estructuras matemáticas son inmutables y eternas" (Tegmark, 2014, p. 318).

Volviendo a la articulación del realismo estructural óntico, encontramos evidencia para sostener que este introduce, sin embargo, la amenaza de la hipótesis del universo matemático. Aunque se sostiene que tenemos que guardar silencio respecto de la distinción entre estructuras matemáticas y estructuras 
físicas, el realismo estructural óntico se limita a mencionar una forma de platonismo matemático que se asume que sería compatible con el principio del cierre naturalista. No se ofrece una caracterización clara de los detalles de tal platonismo matemático. Revisemos este punto a continuación en mayor detalle.

El principio del cierre naturalista dice así:

Cualquier tesis metafísica nueva que tenga que ser considerada con seriedad en el tiempo $t$ debe estar motivada por, y solamente por, el servicio que ella llevaría a cabo, si fuera verdadera, al mostrar cómo dos o más hipótesis científicas específicas, al menos una de las cuales se deriva de la física fundamental, explican conjuntamente más que lo que explican las dos hipótesis tomadas separadamente (Ladyman y Ross, 2007, p. 37).

En este punto, el desafío para el realismo estructural óntico consiste en demostrar que contamos con una forma tal de platonismo matemático que puede, de hecho, satisfacer los requerimientos enunciados en este principio metodológico. No resulta sorprendente que surjan dificultades cuando se observa que las matemáticas no forman parte del enunciado del principio del cierre naturalista. Por el contrario, esta regla metodológica busca esbozar una imagen de la interacción entre la ciencia y la metafísica, según la cual se espera que esta última esté propiamente involucrada en la teorización científica, contribuyendo a la unificación de una o más hipótesis científicas, una de las cuales pertenece a la física fundamental.

Una vía alternativa que podrían seguir los defensores de la tesis del colapso en vistas del principio del cierre naturalista consiste en mantener en pie la apelación al platonismo matemático, pero esta vez argumentando que este tiene que ser concebido como una postura metafísica acerca de la ontología matemática que puede estimular positivamente la unificación de teorías científicas, tal como lo enuncia el principio del cierre naturalista. Aunque esta podría ser una línea atractiva de investigación en metafísica de las ciencias para los defensores del realismo estructural óntico, resulta infructuoso intentar encontrar mayores luces en la elaboración y defensa de esta postura.

Por último, téngase presente que, por un lado, el platonismo matemático ha tomado diversas formas en la discusión reciente, mientras que, por otro, el realismo estructural óntico no aclara cuál de estas formas resulta más adecuada para el principio del cierre naturalista. Dos construcciones estándares del platonismo matemático son las siguientes.

i. Primero, el platonismo matemático irrestricto es la concepción que sostiene que para cada teoría matemática existe una parte del reino matemático que la hace verdadera. Esta concepción se compromete con una ontología 
matemática cabal, que abarca las entidades y las estructuras cuantificadas tanto por las matemáticas puras como por las aplicadas.

ii. En cambio, una segunda versión es el platonismo matemático restringido, ampliamente representado por el platonismo matemático inspirado en Quine y Putnam. Esta versión defiende que para cada teoría matemática exitosamente aplicada a la teorización científica existe una parte del reino matemático que la hace verdadera.

Esta distinción, entre otras variantes del platonismo matemático, puede arrojar luz sobre el problema de si acaso la tesis del colapso arriesga la introducción de la hipótesis del universo matemático, en donde la última se compromete con una forma de pitagoreanismo. Una defensa detallada de la tesis del colapso requiere especificar la forma específica del platonismo matemático que es compatible con el principio del cierre naturalista. Desafortunadamente, no se ofrece tal argumento, aun cuando podría resultar razonable asumir que se piensa en la concepción restringida del platonismo matemático, dada la metafísica naturalista asumida por el realismo estructural óntico.

\section{La concepción inferencial: una respuesta a la tesis del colapso}

En la presente sección se argumenta que podemos evitar la tesis del colapso, rechazando ideas tales como las siguientes: que los límites entre las estructuras matemáticas y las físicas se tornan borrosos en ciertas áreas de la investigación física (French y Ladyman, 2003); que la distinción entre tales estructuras no puede ser trazada porque escapa al principio del cierre naturalista (Ladyman y Ross, 2007); o que el problema de trazar tal distinción representa un pseudoproblema.

En lo que sigue me interesa mostrar que el desafío de distinguir entre estructuras matemáticas y estructuras físicas es un problema genuino en filosofía de las ciencias, filosofía de las matemáticas y metafísica de las ciencias. La literatura reciente nos ofrece diversas estrategias para llevar a cabo esta tarea. Una de ellas consiste en la identificación de estructura matemática excedente en la teorización científica (Redhead, 1975). En ocasiones, de hecho, encontramos más estructura matemática que estructura física en la teorización científica, en donde la estructura matemática excedente nos permite identificar aquellos elementos matemáticos de la representación científica que carecen de interpretación física. La situación al respecto se torna particularmente interesante cuando se observa que tales estructuras matemáticas excedentes juegan un rol epistémico en las teorías de las que ellas forman parte, robusteciendo su poder heurístico, representacional o explicativo, entre otros. 
Tales estructuras matemáticas excedentes carecen de instanciación y de una semántica que las vincule con referentes físicos. Se asume que la instanciación se obtiene a través de la correlación estructural entre rasgos de la estructura matemática y rasgos relevantes de su contraparte física. La estructura física se incorpora en la estructura matemática si logramos dar cuenta de relaciones de mapeo isomórficas (Pincock, 2004) o parcialmente isomórficas u homomórficas (Bueno y French, 2018) entre sus elementos. En contraposición a las estructuras matemáticas, puede indicarse que las estructuras físicas están siempre instanciadas, aun cuando ellas se obtengan a través de procesos de abstracción extensionales (Nguyen y Frigg, 2017) o intensionales.

Volvamos a la distinción entre consideraciones epistémicas y ontológicas. Las consideraciones epistémicas engloban una serie de afirmaciones que enfatizan el rol de las matemáticas en la práctica científica. Las matemáticas parecen ser efectivamente indispensables para la práctica científica, tal como lo sugiere el realismo estructural óntico en su defensa de la tesis del colapso. Destáquese, sin embargo, que la tesis de la indispensabilidad de las matemáticas puede ser leída en clave epistémica, sin involucrar compromisos ontológicos. Desde esta perspectiva, las matemáticas podrían ser indispensables para la teorización en física fundamental, en donde ellas juegan un rol crucial facilitando la construcción de representaciones de estructuras físicas complejas, tales como los estados de entrañamiento cuántico. La indispensabilidad epistémica reconoce, en breve, el hecho de que las matemáticas son una herramienta que fomenta el éxito empírico de las ciencias (Soto, 2019).

Ahora bien, quienes defienden el realismo estructural óntico podrían aceptar la lectura epistémica de la indispensabilidad, pero aun así inclinarse a dar un paso más allá insistiendo en que la única manera de explicar el rol epistémico de las matemáticas en las ciencias físicas consiste en asumir que las estructuras matemáticas existen. En este punto emergerían una vez más las consideraciones ontológicas antes mencionadas: las estructuras matemáticas y las estructuras físicas se encuentran ontológicamente a la par en física fundamental; y los límites que separan las estructuras matemáticas y las físicas desaparecen, forzándonos a adoptar compromisos ontológicos similares con ellas.

La concepción inferencial de la aplicación de estructuras matemáticas a estructuras físicas en la teorización científica nos permite profundizar en las consideraciones epistémicas, arrojando luz sobre algunos de los asuntos que involucra la distinción entre lo matemático y lo físico en las teorías científicas. La concepción inferencial avanza una propuesta metafísicamente deflacionaria acerca del proceso de aplicación de las matemáticas en los siguientes tres pasos: 
i. Inmersión. El primer paso consiste en establecer un mapeo de un sistema físico a partir de una estructura matemática conveniente.

ii. Inferencia. El segundo paso consiste en derivar las consecuencias del formalismo matemático, empleando la estructura matemática obtenida en el paso de la inmersión.

iii. Interpretación. Finalmente, interpretamos las consecuencias matemáticas que se obtienen en el paso de la derivación en términos del sistema físico inicial (Bueno y Colyvan, 2011, p. 353; véase también Bueno y French, 2018, p. 51).

En lo que sigue, analizaré el primer paso relativo al proceso de mapeo de estructuras físicas a partir de estructuras matemáticas. La concepción semántica de las teorías científicas cobra relevancia en este punto. Aunque no puedo desarrollar en todo su detalle tal concepción en el presente artículo, cabe distinguir tres versiones de la concepción semántica. Primero, en un extremo encontramos la versión que apela a relaciones isomórficas entre teorías y modelos (por ejemplo, van Fraassen, 1980), junto a la concepción del mapeo basado en isomorfismos (Pincock, 2004); segundo, en el otro extremo encontramos la versión de la concepción semántica que apela a las relaciones representacionales basadas en la similaridad estructural que instancian los modelos científicos y sus contrapartes físicas (Giere, 1988). Con todo, una tercera opción que ocupa un lugar intermedio y que salva la rigurosidad representacional, a la vez que acomoda las peculiaridades de la representación científica en diversos contextos, es la concepción de las estructuras parciales tal como aparece expuesta en da Costa y French (2003).

La concepción inferencial de la aplicación de las matemáticas funciona dentro del marco de la propuesta de estructuras parciales, la cual sostiene que un modelo de una teoría es cualquier estructura matemática que provee una representación parcial de aspectos relevantes de los fenómenos. De ello se deriva una serie de beneficios que nos permiten trazar distinciones específicas entre estructuras matemáticas y estructuras físicas. Por un lado, la concepción de las estructuras parciales tiene el beneficio de dejar abierta la posibilidad de que nuestro acceso a determinados sistemas físicos sea incompleto, abandonándose la exigencia de ofrecer una correlación isomórfica cabal entre teorías, modelos y sistemas físicos. Desde esta perspectiva, se asume que en diversas ocasiones las representaciones científicas proveen información que refiere selectivamente a ciertos aspectos de sistemas físicos, dejando a un lado información que en ciertos contextos no resulta necesaria para las tareas que se tienen a la vista. Puede haber, en este caso, más estructura física en el objeto que aquella que 
resulta interesante para los propósitos del modelamiento científico. Por otro lado, la concepción de estructuras parciales abandona igualmente la exigencia de proveer una interpretación física completa de las estructuras matemáticas de las teorías, reconociendo el rol que puede jugar en ellas la estructura matemática excedente. Las herramientas matemáticas no interpretadas podrían jugar diversos roles epistémicos en la teorización científica, avanzando caracterizaciones de sistemas inobservables a los que no tenemos acceso epistémico robusto, permitiendo la generación de simulaciones computacionales y modelos, y facilitando la solución de ecuaciones que carecerían de solución sin la estructura matemática adicional.

Aunque solo brevemente, revisemos a continuación la distinción entre estructuras matemáticas y estructuras físicas en vistas del caso de las leyes científicas, el cual resulta particularmente iluminador para nuestro problema si se considera que gran parte de las leyes en física se formulan en términos de estructuras matemáticas que pretenden referir a la estructura física relevante de ciertos sistemas físicos. La decisión de considerar el problema de la distinción entre estructuras físicas y estructuras matemáticas en vistas del caso de las leyes físicas encuentra motivación adicional en ciertos desarrollos recientes del realismo estructural óntico enfocados en leyes, simetrías y modalidad (Berenstain y Ladyman, 2012; French, 2014). Desde esta perspectiva, las leyes son fundamentales en tanto que ellas no supervienen de las relaciones entre propiedades e individuos. Por el contrario, las propiedades y los individuos dependen de las leyes que tienen lugar en la naturaleza y de las relaciones modales (estructurales) que ellas establecen. Aunque por razones adicionales mi concepción de las leyes físicas difiere del fundamentalismo estructural del realismo estructural óntico, encontramos una coincidencia relevante en el uso de la noción de ley científica en términos de enunciados de ley formulados matemáticamente.

Piénsese en el caso conocido, aunque todavía ampliamente debatido, de la ley newtoniana de la gravitación universal (French, 2014, p. 282):

$$
\mathrm{F}_{\text {grav }}=\mathrm{G}\left(\mathrm{m}_{1} \mathrm{~m}_{2}\right) / \mathrm{R}^{2}
$$

La ecuación en cuestión consta de una estructura matemática cuyos elementos se encuentran interpretados, estableciendo relaciones entre las variables $F_{\text {grav }}, G, m_{1}, m_{2}$ y $R^{2}$ cada una de las cuales indexa una cantidad física. Atendiendo al primer paso de la concepción inferencial de la aplicación de las matemáticas, esta ecuación ofrece una estructura matemática conveniente para dar cuenta de la cardinalidad y relaciones estructurales relevantes de sistemas físicos que se 
encuentran afectos a la fuerza de gravedad, tales como dos galaxias, el sistema sol-tierra, dos cuerpos sobre la superficie de la tierra, y otros.

Al llevar a cabo el mapeo entre los nodos de la estructura matemática, por un lado, y la cardinalidad y relaciones estructurales relevantes del sistema físico, por otro, asignamos valores a $m_{1}, m_{2}$ y $R^{2}$. Solamente una vez que se ha establecido una relación de mapeo cabe afirmar que la estructura matemática refiere exitosamente a una instancia determinada de estructura física. Aunque el caso en cuestión sea tomado de la mecánica clásica, este nos permite destacar que ninguno de los elementos indicados requiere eliminar las fronteras entre las estructuras físicas y las matemáticas. No se suscitan problemas metafísicos con respecto a la posible fusión de lo abstracto y lo concreto, abandonándose cualquier motivación que promueva la tesis del colapso o la hipótesis del universo matemático.

Desde una perspectiva histórica, la ecuación newtoniana de la gravitación universal nos enseña algo acerca de la evolución teórica de aquellos elementos de la estructura matemática que contaron en algún momento como excedentes. En sus orígenes en el tiempo de Newton, la constante $G$ carecía de interpretación física empíricamente fundada. El valor aproximado de $G$ no se obtuvo entonces a partir de experimentos midiendo la fuerza gravitacional, sino solo a partir de las soluciones requeridas por la ecuación para salvar los fenómenos observados. Tal constante parece ocupar el lugar de un elemento matemático excedente en la estructura general, el cual, a pesar de carecer de un referente físico, era necesario para llevar a cabo predicciones y explicaciones de fenómenos gravitacionales. Solamente más tarde, los experimentos de Cavendish dieron a conocer un valor físico aproximado para $G$ (a saber, $6.754 \times 10^{-11} \mathrm{Nm} 2 / \mathrm{kg} 2$, en unidades actuales MKS), proveyendo una interpretación física para lo que era un supuesto matemático orientado a facilitar soluciones físicamente adecuadas de la ecuación.

El análisis llevado a cabo permite arrojar luz sobre los siguientes puntos:

i. Las estructuras matemáticas se emplean para representar estructuras de sistemas físicos. Una propuesta acerca de cómo se lleva a cabo tal relación representacional es ofrecida por la teoría del mapeo dentro de la concepción semántica.

ii. Dentro de esa última, la aproximación de las estructuras parciales que opera de trasfondo a la concepción inferencial de la aplicación de las matemáticas nos permite entender la aplicación de estructuras matemáticas a estructuras físicas en términos de relaciones parcialmente isomórficas u homomórficas.

iii. Algunos elementos de las estructuras matemáticas son excedentes, vale decir, no cuentan con interpretación física, ni pueden mucho menos 
ser identificados con estructuras físicas. Sin embargo, tales estructuras matemáticas excedentes pueden jugar roles cruciales en la teorización científica en diversos casos, fomentando el poder epistémico de las teorías.

iv. La estructura física corresponde a la cardinalidad y a las relaciones estructurales relevantes que se identifican en sistemas físicos de interés en diversos contextos de investigación. Tal cardinalidad y relaciones estructurales son mapeadas por los nodos y las relaciones entre ellos en la teoría matemática, tales como las variables, funciones, o constantes, entre otras.

\section{Observaciones finales}

En este artículo se ha examinado críticamente la tesis de colapso de las estructuras físicas y matemáticas propuesta por el realismo estructural óntico. A partir de una distinción entre consideraciones epistémicas y ontológicas, y asumiendo algunas de las herramientas de la concepción inferencial de la aplicación de estructuras matemáticas a estructuras físicas, he mostrado que no necesitamos adoptar la tesis del colapso, ni las afirmaciones que ella involucra. Entre las últimas, cabe abandonar ideas tales como que no hay distinción entre lo abstracto o lo concreto; o que los límites entre las estructuras matemáticas y las físicas se tornan borrosos o simplemente desaparecen; o que las estructuras matemáticas y físicas se encuentran ontológicamente a la par.

Aun cuando podamos aceptar que las matemáticas son ampliamente indispensables en física fundamental, tal indispensabilidad no hace necesario que eliminemos los límites entre las estructuras matemáticas y las físicas. Igualmente, ello no requiere que se adopte la hipótesis del universo matemático. Por el contrario, la concepción inferencial de la aplicación de las matemáticas a las ciencias físicas arroja luz sobre consideraciones epistémicas acerca del lugar y del rol de las estructuras matemáticas y físicas en la teorización científica. Algunas estructuras matemáticas resultan adecuadas para representar la estructura de sistemas físicos; otras estructuras matemáticas contienen elementos excedentes, los cuales resultan imprescindibles a pesar de carecer de interpretación física en una determinada etapa de la investigación científica; las estructuras físicas - a diferencia de las matemáticas - están determinadas por la relación entre los elementos relevantes del sistema físico, los cuales a su vez son incorporados en los nodos de las estructuras matemáticas (tales como las variables, las funciones, o las constantes, entre otros) que resulten convenientes en vistas de casos específicos. Cabe concluir que nada de esto sugiere la tesis del colapso, ni mucho menos la hipótesis del universo matemático. Se pueden trazar distinciones relevantes entre estructuras físicas y matemáticas sin hacer de ello un problema metafísico colosal. 
Tengo presente que el análisis de la distinción entre estructuras físicas y estructuras matemáticas en el caso de las leyes físicas desde la perspectiva de la concepción inferencial tiene un alcance limitado (sección 4). Diversas observaciones ponen en cuestión este desarrollo. Primero, la matemática se aplica a las ciencias en diversos escenarios, y el caso de las leyes científicas es solo uno de ellos. Segundo, la concepción inferencial podría funcionar de manera diferente para distintas leyes, en vistas de las peculiaridades de cada una de estas últimas, tales como la extensión de su estructura matemática excedente o la disponibilidad de valores físicos determinados para las variables que constituyen la estructura matemática que expresa la ley. Y tercero, hasta ahora solamente me he referido al caso de la ley de gravitación universal newtoniana, a sabiendas de que hay casos quizás más interesantes de estructura matemática excedente en teorías de la mecánica cuántica, tales como el empleo de los espacios de Hilbert.

\section{Referencias}

BERENSTAIN, N., J. LADYMAN. "Ontic structural realism and modality”. (pp. 149168). In: Structural Realism: Structure, Object, and Causality. Eds. E. M. Landry and D. P. Rickles. Dordrecht: Springer, 2012.

BUENO, O., COLYVAN, M. "An Inferential Conception of the Application of Mathematics”, Nous, Vol. 45, Nr. 2, pp. 345-374, 2011.

BUENO, O., FRENCH, S. “Applying Mathematics. Immersion, Inference, Interpretation”. Oxford: Oxford University Press, 2018.

CHIAPPIN, J. R. N. "Duhem's Theory of Science: An Interplay between History and Philosophy of Science". Disertación Doctoral. Pittsburgh: University of Pittsburgh, 1989. CHIAPPIN, J. R. N. "A Methodology for the Theory of Science and Its Application to the Reconstruction of the Metaphysical Level of Duhem's Theory of Science". Revista Portuguesa de Filosofia, Vol. 74, Nr. 1, pp. 19-58, 2018.

COLYVAN, M. "The Indispensability of Mathematics". Oxford: Oxford University Press, 2001.

DA COSTA, N., FRENCH, S. "Science and Partial Truth: A Unitary Approach to Models and Scientific Reasoning”. Oxford, Oxford University Press, 2003.

FEYNMAN, R. "The Character of a Physical Law". London: Penguin Books, 1965.

FIELD, H. "Science without Numbers. A Defence of Nominalism". Princeton: Princeton University Press, 1980.

FIELD, H. "Realism, Mathematics and Modality". New York, Basil Blackwell, 1989. FRENCH, S. "The Structure of the World. Metaphysics and Representation". Oxford: Oxford University Press, 2014.

FRENCH, S., LADYMAN, J. "The dissolution of objects: between platonism and phenomenalism”. Synthese, Vol. 136, Nr. 1, pp. 73-77, 2003. 
FRENCH, S., LADYMAN, J. "In defence of ontic structural realism". (pp. 25-42). In: Scientific Structuralism. Eds. A. Bokulich y P. Bokulich. Dordrecht: Springer, 2011. GIERE, R. "Explaining Science. A Cognitive Approach". Chicago and London, The University of Chicago Press, 1988.

KYLE STANFORD, P., HUMPHREYS, P., HAWLEY, K., LADYMAN, J., ROSS, D. "Protecting rainforest realism". Metascience: An International Review Journal for the History, Philosophy and Social Studies of Science, Vol. 19, Nr. 2, pp. 161-185, 2010. LADYMAN, J. "What is structural realism?". Studies in the History and the Philosophy of Science, Part A, Vol. 29, Nr. 3, pp. 409-424, 1998.

LADYMAN, J. “An apology for naturalized metaphysics”. (pp. 141-161). In: Metaphysics and the Philosophy of Science. New Essays. Eds. M. Slater y Z. Yudell. Oxford: Oxford University Press, 2017.

LADYMAN, J., ROSS, D. "The world in the data". (pp. 108-150). In: Scientific Metaphysics. Eds. D. Ross, J. Ladyman y H. Kincaid. Oxford: Oxford University Press, 2013.

LADYMAN, J., ROSS, D., SPURRET, con D., COLLIER, J. "Every Thing Must Go. Metaphysics Naturalized". Oxford: Oxford University Press, 2007.

NGUYEN, J., FRIGG, R. "Mathematics Is Not the Only Language in the Book of Nature", Synthese, https://doi.org/10.1007/s11229-017-1526-5, 2017.

PINCOCK, C. "A New Perspective on the Problem of Applying Mathematics", Philosophia Mathematica 3(12), pp. 135-161., 2004.

PINCOCK, C. "Mathematical Structural Realism". (pp. 67-79). In: Scientific Structuralism. Eds, A. Bokulich y P. Bokulich. Dordrecht: Springer, 2011.

PSILLOS, S. "Scientific Realism: How Science Tracks Truth". New York: Routledge, 1999.

READHEAD, M. "Symmetry in Intertheoretical Relations", Synthese, 32, pp. 77-112, 1975.

ROSS, D., LADYMAN, J., KINCAID, H. (eds.). "Scientific Metaphysics". Oxford: Oxford University Press, 2013.

SOTO, C. "The Epistemic Indispensability Argument". Journal for General Philosophy of Science. pp. 1-17, 2019. https://doi.org/10.1007/s10838-018-9437-9.

TEGMARK, M. "The mathematical universe". Foundations of Physics, Nr. 38, pp. 101-150, 2008.

TEGMARK, M. "Our Mathematical Universe. My Quest for the Ultimate Nature of Reality”. London: Penguin Books, 2014.

VAN FRAASSEN, B. “The Scientific Image". Oxford: Clarendon Press, 1980.

WORRALL, J. "Structural Realism: The Best of Both Worlds?". Dialectica, Vol. 43, Nr. 1-2, pp. 99-124, 1989. 